This Section of Epidemiology and Psychiatric Sciences appears in each issue of the Journal to stress the relevance of epidemiology for behavioral neurosciences, reporting the results of studies that explore the use of an epidemiological approach to provide a better understanding of the neural basis of major psychiatric disorders and, in turn, the utilisation of the behavioural neurosciences for promoting innovative epidemiological research.

The ultimate aim is to help the translation of most relevant research findings into every-day clinical practice. These contributions are written in house by the journal's editorial team or commissioned by the Section Editor (no more than 1000 words, short unstructured abstract, 4 key-words, one Table or Figure and up to ten references).

Paolo Brambilla, Section Editor

\title{
Metabolic alterations in generalised anxiety disorder: a review of proton magnetic resonance spectroscopic studies
}

\author{
G. Delvecchio ${ }^{1}$, J. A. Stanley ${ }^{2}$, A. C. Altamura ${ }^{1}$ and P. Brambilla ${ }^{1,3 *}$ \\ ${ }^{1}$ Department of Neurosciences and Mental Health, Fondazione IRCCS Ca' Granda Ospedale Maggiore Policlinico, University of Milan, Milan, Italy \\ ${ }^{2}$ Department of Psychiatry and Behavioral Neurosciences, Wayne State University School of Medicine, Detroit, MI, USA \\ ${ }^{3}$ Department of Psychiatry and Behavioural Neurosciences, University of Texas at Houston, TX, USA
}

\begin{abstract}
Generalised anxiety disorder (GAD) is a common psychiatric illness characterised by selective morpho-functional brain alterations. The breath of neuroimaging studies investigating the neural basis of GAD is extensive; however, its pathophysiology is still largely unknown. Specifically for proton Magnetic Resonance Spectroscopy ( ${ }^{1} \mathrm{H}$ MRS) investigations, which have the aim of identifying differences in metabolite levels between conditions in key brain areas, often showed contrasting results. Indeed, there are selected ${ }^{1} \mathrm{H}$ MRS studies reporting deficits of key metabolites in GAD patients; however, collectively the literature remains mixed with respect to consistency of major findings. In this review, we evaluate published ${ }^{1} \mathrm{H}$ MRS studies on GAD with the final aim of providing a comprehensive overview of the extent of neurometabolic dysfunctions associated with GAD. Interestingly, the majority of the studies reviewed showed altered metabolite levels in the dorsolateral prefrontal cortex and hippocampus suggesting regional specificity. These results also provide evidence of the utility of ${ }^{1} \mathrm{H}$ MRS not only for elucidating the pathophysiology of neuropsychiatric diseases, but also for the identification of more beneficial and targeted pharmacological interventions. Additionally, future studies are warranted to overcome methodological differences observed across the studies.
\end{abstract}

Received 14 June 2017; Accepted 21 June 2017; First published online 9 August 2017

Key words: Generalised anxiety disorder, magnetic resonance spectroscopy, metabolites, neurochemicals.

Generalised anxiety disorder (GAD) is a common psychiatric disease characterised by specific physical and psychological symptoms, including persisting worry, irritability and fatigue (DSM5, American Psychiatry Association, 2013; Diwadkar et al. 2017). GAD causes high human suffering, which is poorly understood.

\footnotetext{
* Address for correspondence: P. Brambilla, Department of Neurosciences and Mental Health, Fondazione IRCCS $\mathrm{Ca}^{\prime}$ Granda Ospedale Maggiore Policlinico, via F. Sforza 35, 20122 Milan, Italy.

(Email: paolo.brambilla1@unimi.it)
}

With respect to neuroimaging studies, the exploration of putative biomarkers of this disease is still at an early stage (Terlevic et al. 2012). This is true especially for the application of proton Magnetic Resonance Spectroscopy ( ${ }^{1} \mathrm{H}$ MRS), which has the unique ability of providing important quantitative biochemical information in localised brain areas (Stanley, 2002). This can lead to identifying possible and more effective pharmacological treatments for GAD. The prominent ${ }^{1} \mathrm{H}$ metabolites include $\mathrm{N}$-acetyl-aspartate (NAA), a marker for neuronal density and functioning, 
glycerophosphocholine plus phosphocholine (GPC+ PC), membrane phospholipid metabolites, and phosphocreatine and creatine $(\mathrm{PCr}+\mathrm{Cr})$, involved in energetic processes (Brambilla et al. 2002, 2012; Stanley et al. 2007).

In this review, we aimed at providing an overview of ${ }^{1} \mathrm{H}$ MRS studies carried out in GAD with the final goal of shedding light on the significance of the reported altered metabolite levels in this disorder. A bibliographic search on PUBMED on ${ }^{1} \mathrm{H}$ MRS studies in GAD was performed and the research terms used were 'MRS', 'spectroscopy', and 'generalised anxiety disorder'. Studies were excluded if the publications: (a) included twin samples, (b) investigated GAD not in relation to healthy controls $(\mathrm{HC})$ or (c) explored only white matter structures. In total, eleven papers met the inclusion criteria and are summarised in Table 1. Briefly, among the 11 studies retrieved, the majority employed a multi-voxel $(N=7)$ instead of a single-voxel $(N=4){ }^{1} \mathrm{H}$ MRS technique and a $1.5 \mathrm{~T}$ scanner $(N=6)$ instead of a $3 \mathrm{~T}(N=4)$ or a $4 \mathrm{~T}(N=1)$ scanner. Interestingly, all the ${ }^{1} \mathrm{H}$ MRS studies on GAD, except for two studies Abdallah et al. 2012a; Strawn et al. 2013), focused on brain regions within the hippocampus and dorsolateral prefrontal cortex (DLPFC).

Regarding the DLPFC, four studies of which three of them are from the same group, reported multiple contrasts including higher ratios of $\mathrm{NAA} / \mathrm{PCr}+\mathrm{Cr}$ (Mathew et al. 2004) and lower ratios of GPC + PC/ $\mathrm{PCr}+\mathrm{Cr}$ and GPC + PC/NAA (Moon et al. 2015; 2016b; Moon \& Jeong, 2016a) ratios in GAD patients compared with HC. Additionally, Raparia et al. (2016) found higher NAA, PCr $+\mathrm{Cr}$ and $\mathrm{GPC}+\mathrm{PC}$ levels in DLPFC, premotor cortex (PC) and secondary somatosensory cortex (SSC) bilaterally in GAD patients compared with HC. Interestingly, Mathew et al. (2004) reported that GAD patients with childhood abuse had higher NAA/PCr $+\mathrm{Cr}$ ratios compared with GAD patients without childhood abuse. In contrast, Raparia et al. (2016) found that GAD patients had no significant associations between emotional abuse scores and NAA, $\mathrm{PCr}+\mathrm{Cr}$ and $\mathrm{GPC}+\mathrm{PC}$ levels in DLPFC, PC and SSC bilaterally, but were significant in HC. Additionally, the three studies carried out by Moon et al. found that GPC + PC/PCr $+\mathrm{Cr}$ and GPC + $\mathrm{PC} / \mathrm{NAA}$ ratios positively correlated with right DLPFC volumes (Moon \& Jeong, 2016a; Moon et al. 2016b) and blood oxygenation level-dependent signal change in right DLPFC (Moon et al. 2016b). In contrast, a negative correlation was observed between GPC + PC/NAA ratio and anxiety symptom severity (Moon et al. 2015). Collectively, these studies suggest DLPFC neuronal deficits, which may in turn explain the neurocognitive deficits often observed in GAD patients. Indeed DLPFC is a key brain area regulating cognition and emotion, and plays a prominent role in working memory and executive brain functions (Brambilla et al. 2005).

Regarding the hippocampus, the study by Mathew et al. (2008) showed increased hippocampal NAA levels after 8 weeks of treatment with the glutamateantagonist riluzole in GAD responder patients, whereas hippocampal NAA levels decreased over time in nonresponders. Moreover, the change over time (postminus pre treatment) in hippocampal volume was positively associated with change over time in NAA (especially in the right side) and with the improvement in anxiety symptoms (Abdallah et al. 2013). In contrast, lower ratios of NAA/PCr $+\mathrm{Cr}$ in bilateral hippocampus of nine GAD patients were not reversed after 12 weeks of paroxetine, despite marked symptoms improvement (Mathew et al. 2010). Additionally, Abdallah et al. (2012a) observed a negative correlation between right occipital NAA and symptoms improvement after riluzole treatment. Riluzole has been demonstrated to modulate extracellular glutamate through glial reuptake mechanisms regulating neural plasticity in the hippocampus (Frizzo et al. 2004). SSRIs have also been linked to enhance neural plasticity in hippocampal cells (Wang et al. 2008). Therefore, hippocampal NAA may reflect non-neuronal activity (Mathew et al. 2008) being a possible biomarker of GAD, and NAA change might be differently related to disparate mechanisms of drug action. Additionally, Coplan et al. (2014) also reported significant metabolites alterations associated with weight, with overweight GAD patients showing lower NAA in hippocampus compared with HC. Moreover, an inverse correlation was observed between hippocampal NAA and body mass index as well as higher worry predicted low hippocampal NAA and $\mathrm{PCr}+\mathrm{Cr}$. Lastly, Strawn et al. (2013) recently reported no significant alterations in glutamate $/ \mathrm{PCr}+\mathrm{Cr}$ ratios in the anterior cingulate of adolescents with GAD; however, a positive correlations between glutamate/PCr $+\mathrm{Cr}$ and anxiety symptoms severity.

In conclusions, these findings together suggest that GAD is associated with metabolic dysfunctions in selective brain regions, including the DLPFC and hippocampus. However, these results require further independent replications. Indeed, although the majority of the studies employed absolute metabolite values, some others used metabolite ratios, which might have therefore limited the interpretations of the results. Additionally, most of the studies were characterised by relatively small sample sizes and were carried out by the same research group, further decreasing the generalisability of their findings. Despite these limitations, these findings illustrate that alterations in specific metabolites, especially NAA, PCr $+\mathrm{Cr}$ and GPC $+\mathrm{PC}$, might be considered putative biomarkers of 
Table 1. Selection of studies on generalised anxiety disorder exploring metabolic alterations with 1-H magnetic resonance spectroscopy

\begin{tabular}{|c|c|c|c|c|c|c|c|c|}
\hline Study & $\begin{array}{c}\text { Sample (age, } \\
\text { mean } \pm \text { S.D.) }\end{array}$ & Gender F/M & Study design & $\begin{array}{l}\text { Psychotropic } \\
\text { medications }\end{array}$ & Method & $\begin{array}{l}\text { Location (voxel } \\
\text { size) }\end{array}$ & $\begin{array}{l}\text { Quantification } \\
\text { and reported }{ }^{1} \mathrm{H} \\
\text { metabolites }\end{array}$ & ${ }^{1} \mathrm{H}$ MRS findings \\
\hline $\begin{array}{l}\text { Mathew } \\
\text { et al. } \\
\text { (2004) }\end{array}$ & $\begin{array}{l}\text { GAD patients }= \\
15(39.3 \pm 13.3) \\
\text { HC }=15(39.1 \pm 13.5)\end{array}$ & $\begin{array}{l}\text { GAD patients }=8 / 7 \\
\mathrm{HC}=8 / 7\end{array}$ & Cross-sectional & $\begin{array}{l}\text { Six medication-naïve. } \\
\text { No psychotropic drugs } \\
\text { within at least } 4 \\
\text { weeks }\end{array}$ & $\begin{array}{l}\text { Multi-voxel }{ }^{1} \mathrm{H} \\
\text { MRSI with } \\
\mathrm{TE}=280 \mathrm{~ms} \\
\text { at } 1.5 \mathrm{~T}\end{array}$ & $\begin{array}{l}\text { Left and right } \\
\text { hippocampus } \\
\text { and DLPFC } \\
(1.5 \times 0.75 \times \\
\left.0.75 \mathrm{~cm}^{3}\right)\end{array}$ & $\begin{array}{l}\text { Metabolite ratio } \\
\mathrm{NAA} / \mathrm{PC}+\mathrm{Cr} \\
\mathrm{GPC}+\mathrm{PC}\end{array}$ & $\begin{array}{l}\text { - Higher NAA } \backslash \text { Cr ratio in } \\
\text { right DLPFC in GAD } \\
\text { patients compared with HC. } \\
\text { - GAD patients with } \\
\text { childhood abuse had higher } \\
\text { NAA } \backslash \text { creatine ratio in right } \\
\text { DLPFC compared with } \\
\text { GAD patients without } \\
\text { childhood abuse }\end{array}$ \\
\hline $\begin{array}{l}\text { Mathew } \\
\text { et al. } \\
(2008)\end{array}$ & $\begin{array}{l}\text { GAD patients }= \\
14(31.7 \pm 9.6) \\
\mathrm{HC}=7(27.4 \pm 4.2)\end{array}$ & $\begin{array}{l}\text { GAD patients }=8 / 6 \\
\mathrm{HC}=5 / 2\end{array}$ & Longitudinal & $\begin{array}{l}\text { Six medication- naïve. } \\
\text { No current } \\
\text { psychotropic drugs }\end{array}$ & $\begin{array}{l}\text { Multi-voxel }{ }^{1} \mathrm{H} \\
\text { MRSI with } \\
\mathrm{TE}=280 \mathrm{~ms} \\
\text { at } 1.5 \mathrm{~T} \text {. } \\
8 \text { weeks of } \\
\text { therapy with } \\
\text { Riluzole }\end{array}$ & $\begin{array}{l}\text { Left and right } \\
\text { hippocampus } \\
\left(1.13 \mathrm{~cm}^{3}\right)\end{array}$ & $\begin{array}{l}\text { Absolute relative } \\
\text { to water } \\
\text { NAA } \\
\text { PCr+Cr } \\
\text { GPC+PC }\end{array}$ & $\begin{array}{l}\text { - Increased NAA in } \\
\text { hippocampus bilaterally, in } \\
\text { responder patients over } \\
\text { time. } \\
\text { - Decrease NAA in } \\
\text { hippocampus bilaterally } \\
\text { over time in non- } \\
\text { responders patients. } \\
\text { - No differences in NAA } \\
\text { concentrations between } \\
\text { responders and non- } \\
\text { responders patients in any } \\
\text { time point. } \\
\text { - In GAD patients, } \\
\text { hippocampal NAA } \\
\text { concentration and } \\
\text { proportional increase in } \\
\text { NAA from baseline were } \\
\text { positively associated with } \\
\text { improvements in worry and } \\
\text { clinician-rated anxiety }\end{array}$ \\
\hline $\begin{array}{l}\text { Abdallah } \\
\text { et al. } \\
(2012 a)\end{array}$ & $\begin{array}{l}\text { GAD patients }=14(33.9 \\
\quad \pm 2.7) \\
\mathrm{HC}=10(30.3 \pm 2.4)\end{array}$ & $\begin{array}{l}\text { GAD patients }=8 / 6 \\
\mathrm{HC}=6 / 4\end{array}$ & Longitudinal & $\begin{array}{l}\text { Fourteen medication- } \\
\text { free }\end{array}$ & $\begin{array}{l}\text { Multi-voxel }{ }^{1} \mathrm{H} \\
\text { MRSI with } \\
\text { TE }=280 \mathrm{~ms}\end{array}$ & $\begin{array}{l}\text { Left and right } \\
\text { lateral }\end{array}$ & $\begin{array}{c}\text { Absolute relative } \\
\text { to water NAA }\end{array}$ & $\begin{array}{l}\text { - Negative correlation } \\
\text { between right occipital } \\
\text { NAA and symptoms }\end{array}$ \\
\hline
\end{tabular}




\begin{tabular}{|c|c|c|c|c|c|c|c|c|}
\hline Study & $\begin{array}{c}\text { Sample (age, } \\
\text { mean } \pm \text { S.D.) }\end{array}$ & Gender F/M & Study design & $\begin{array}{l}\text { Psychotropic } \\
\text { medications }\end{array}$ & Method & $\begin{array}{l}\text { Location (voxel } \\
\text { size) }\end{array}$ & $\begin{array}{l}\text { Quantification } \\
\text { and reported }{ }^{1} \mathrm{H} \\
\text { metabolites }\end{array}$ & ${ }^{1} \mathrm{H}$ MRS findings \\
\hline & & & & & $\begin{array}{l}\text { and MRI at } \\
1.5 \mathrm{~T} \text {. } \\
8 \text { weeks of } \\
\text { therapy with } \\
\text { Riluzole }\end{array}$ & $\begin{array}{l}\text { occipital } \\
\left(1.13 \mathrm{~cm}^{3}\right)\end{array}$ & & $\begin{array}{l}\text { improvement after } \\
\text { treatment }\end{array}$ \\
\hline $\begin{array}{l}\text { Abdallah } \\
\quad \text { et al. } \\
(2012 b)\end{array}$ & $\begin{array}{l}\text { GAD patients }=18(33.9 \\
\quad \pm 2.7) \\
\mathrm{HC}=10(30.3 \pm 2.4)\end{array}$ & $\begin{array}{l}\text { GAD patients }=8 / 10 \\
\mathrm{HC}=6 / 4\end{array}$ & Longitudinal & $\begin{array}{l}\text { Eighteen medication- } \\
\text { free }\end{array}$ & $\begin{array}{l}\text { Multi-voxel }{ }^{1} \mathrm{H} \\
\text { MRSI with } \\
\mathrm{TE}=280 \mathrm{~ms} \\
\text { and MRI at } \\
1.5 \mathrm{~T} \text {. } \\
8 \text { weeks of } \\
\text { therapy with } \\
\text { Riluzole }\end{array}$ & $\begin{array}{l}\text { Left and right } \\
\text { hippocampus } \\
\left(1.13 \mathrm{~cm}^{3}\right)\end{array}$ & $\begin{array}{l}\text { Absolute relative } \\
\text { to water NAA }\end{array}$ & $\begin{array}{l}\text { - Reduction in total } \\
\text { hippocampal volume at } \\
\text { baseline in GAD patients } \\
\text { (more pronounced in } \\
\text { remitters). } \\
\text { - Delta (final-baseline) } \\
\text { ippocampal volume } \\
\text { positively correlate with } \\
\text { delta NAA (especially on } \\
\text { the right side) in GAD. }\end{array}$ \\
\hline $\begin{array}{l}\text { Mathew } \\
\text { et al. } \\
\text { (2010) }\end{array}$ & $\begin{array}{l}\text { GAD patients }=9(41.7 \\
\quad \pm 15.8) \\
\text { HC }=10(37.1 \pm 14.8)\end{array}$ & $\begin{array}{l}\text { GAD patients }=4 / 5 \\
\mathrm{HC}=4 / 10\end{array}$ & Longitudinal & $\begin{array}{l}\text { Four medication-naïve. } \\
\text { No psychotropic drugs } \\
\text { within at least } 4 \\
\text { weeks }\end{array}$ & $\begin{array}{l}\text { Multi-voxel }{ }^{1} \mathrm{H} \\
\text { MRSI with } \\
\mathrm{TE}=280 \mathrm{~ms} \\
\text { at } 1.5 \mathrm{~T} . \\
12 \text { weeks of } \\
\text { therapy with } \\
\text { Paroxetine }\end{array}$ & $\begin{array}{l}\text { Left and right } \\
\text { hippocampus } \\
(1.5 \times 0.75 \times \\
0.75 \mathrm{~cm}^{3} \text { or } \\
0.84 \mathrm{cc})\end{array}$ & $\begin{array}{l}\text { Metabolite ratio } \\
\text { NAA/PCr+Cr }\end{array}$ & $\begin{array}{l}\text { - Lower levels of NAA/Cr } \\
\text { ratio in bilateral } \\
\text { hippocampus in GAD } \\
\text { patients compared with } \\
\text { HC, before and after } \\
\text { therapy. } \\
\text { - Hippocampal NAA/Cr } \\
\text { ratios were positively } \\
\text { correlated with PSWQ }\end{array}$ \\
\hline $\begin{array}{l}\text { Strawn } \\
\text { et al. } \\
\text { (2013) }\end{array}$ & $\begin{array}{l}\text { GAD patients }=10(14 \pm \\
2.2) \\
\mathrm{HC}=10(14.5 \pm 2.3)\end{array}$ & $\begin{array}{l}\text { GAD patients }=6 / 4 \\
\mathrm{HC}=6 / 4\end{array}$ & Cross-sectional & $\begin{array}{l}\text { No psychotropic drugs } \\
\text { within at least five } \\
\text { half-lives }\end{array}$ & $\begin{array}{l}\text { Single-voxel } \\
{ }^{1} \mathrm{H}-\mathrm{MRS} \\
\text { with TE }=30 \\
\mathrm{~ms} \text { at } 4 \mathrm{~T}\end{array}$ & $\begin{array}{l}\operatorname{ACC}(2.2 \times 2.2 \times \\
\left.2.2 \mathrm{~cm}^{3}\right)\end{array}$ & $\begin{array}{l}\text { Metabolite ratio } \\
\mathrm{Glu} / \mathrm{PCr}+\mathrm{Cr}\end{array}$ & $\begin{array}{l}\text { - No differences in NAA, } \\
\text { creatine or myo-inositol } \\
\text { between GAD and HC. } \\
\text { - Positive correlations } \\
\text { between Glu/PCr + Cr and } \\
\text { anxiety symptom severity }\end{array}$ \\
\hline $\begin{array}{l}\text { Raparia } \\
\text { et al. } \\
\text { (2016) }\end{array}$ & $\begin{array}{l}\text { GAD patients }= \\
16(37.9 \pm 14.2) \\
\mathrm{HC}=16(35.3 \pm 10.3)\end{array}$ & $\begin{array}{l}\text { GAD patients }=11 / 5 \\
\mathrm{HC}=10 / 6\end{array}$ & Cross-sectional & $\begin{array}{l}\text { No medication for at } \\
\text { least } 2 \text { weeks prior } \\
\text { the MRSI scan }\end{array}$ & $\begin{array}{l}\text { Multi-voxel } \\
{ }^{1} \mathrm{H}-\mathrm{MRSI} \\
\text { with TE }= \\
280 \mathrm{~ms} \text { at } 3 \mathrm{~T}\end{array}$ & $\begin{array}{l}\text { mPFC PMC SCC } \\
\quad(7.5 \times 7.5 \times \\
\left.15 \mathrm{~mm}^{3}\right)\end{array}$ & $\begin{array}{l}\text { Absolute relative } \\
\text { to water NAA } \\
\mathrm{PCr}+\mathrm{Cr} \\
\mathrm{GPC}+\mathrm{PC}\end{array}$ & $\begin{array}{l}\text { - In GAD patients, emotional } \\
\text { abuse scores did not } \\
\text { correlate with either NAA, } \\
\text { Cr or Cho levels for the }\end{array}$ \\
\hline
\end{tabular}


Table 1. Continued

\begin{tabular}{|c|c|c|c|c|c|c|c|c|}
\hline Study & $\begin{array}{c}\text { Sample (age, } \\
\text { mean } \pm \text { S.D.) }\end{array}$ & Gender F/M & Study design & $\begin{array}{c}\text { Psychotropic } \\
\text { medications }\end{array}$ & Method & $\begin{array}{l}\text { Location (voxel } \\
\text { size) }\end{array}$ & $\begin{array}{l}\text { Quantification } \\
\text { and reported }{ }^{1} \mathrm{H} \\
\text { metabolites }\end{array}$ & ${ }^{1} \mathrm{H}$ MRS findings \\
\hline & & & & & & & & $\begin{array}{l}\text { mPFC, PMC and SCC } \\
\text { bilaterally. } \\
\text { - GAD patients exhibited } \\
\text { greater NAA, Cr and Cho } \\
\text { than HC. } \\
\text { - CTQ emotional abuse effect } \\
\text { was inversely predicting } \\
\text { NAA, Cr and Cho only in } \\
\text { HC }\end{array}$ \\
\hline $\begin{array}{l}\text { Moon \& } \\
\text { Jeong } \\
(2016 a)\end{array}$ & $\begin{array}{l}\text { GAD patients }= \\
14(36.6 \pm 8.8) \\
\mathrm{HC}=14(37.8 \pm 7.8)\end{array}$ & $\begin{array}{l}\text { GAD patients }=8 / 6 \\
\mathrm{HC}=8 / 6\end{array}$ & Cross-sectional & $\begin{array}{l}\text { Eleven patients with } \\
\text { Anxiolytics and/or } \\
\text { antidepressants. } \\
\text { Three patients with } \\
\text { single psychiatric } \\
\text { medication } \\
\text { comprising } \\
\text { escitalopram or } \\
\text { bupropion }\end{array}$ & $\begin{array}{l}\text { Single-voxel } \\
{ }^{1} \mathrm{H}-\mathrm{MRS} \\
\text { with TE }=30 \\
\mathrm{~ms} \text { and MRI } \\
\text { at } 3 \mathrm{~T}\end{array}$ & $\begin{array}{l}\text { DLPFC }(20 \times \\
20 \times 20 \text { or } \\
\left.8 \mathrm{~cm}^{3}\right)\end{array}$ & $\begin{array}{l}\text { Metabolite ratio } \\
\text { NAA } \\
\text { PCr }+ \text { Cr } \\
\text { GPC }+ \text { PC } \\
\text { Ml } \\
\text { Lactate } \\
\text { Lip } \\
\alpha-G l x / P C r+C r \\
\beta, \gamma-G l x / N A A\end{array}$ & $\begin{array}{l}\text { - GAD patients had } \\
\text { significantly lower Cho/Cr } \\
\text { and Cho/NAA ratios in the } \\
\text { DLPFC compared with } \\
\text { HC. } \\
\text { - DLPFC volume was } \\
\text { positively correlated with } \\
\text { the ratios of Cho/Cr and } \\
\text { Cho/NAA in GAD patients }\end{array}$ \\
\hline $\begin{array}{l}\text { Moon } \\
\text { et al. } \\
(2016 b)\end{array}$ & $\begin{array}{l}\text { GAD patients }=13(37.8 \\
\quad \pm 7.6) \\
\mathrm{HC}=13(35.9 \pm 3.5)\end{array}$ & $\begin{array}{l}\text { GAD patients }=7 / 6 \\
\mathrm{HC}=7 / 6\end{array}$ & Cross-sectional & $\begin{array}{l}\text { Seven patients with } \\
\text { Anxiolytics and/or } \\
\text { antidepressants. } \\
\text { Six patients each were } \\
\text { taking one } \\
\text { psychotropic } \\
\text { medication }\end{array}$ & $\begin{array}{l}\text { Single-voxel } \\
{ }^{1} \mathrm{H}-\mathrm{MRS} \\
\text { with TE }=30 \\
\mathrm{~ms} \text { and } \mathrm{fMRI} \\
\text { at } 3 \mathrm{~T}\end{array}$ & $\begin{array}{l}\text { DLPFC }(20 \times \\
20 \times 20 \text { or } \\
\left.8 \mathrm{~cm}^{3}\right)\end{array}$ & $\begin{array}{l}\text { Metabolite ratio } \\
\alpha-\mathrm{Glx} / \mathrm{PCr}+\mathrm{Cr} \\
\mathrm{mI} / \mathrm{PCr}+\mathrm{Cr} \\
\mathrm{GPC}+\mathrm{PC} / \mathrm{PCr}+ \\
\quad \mathrm{Cr} \\
\beta, \gamma-\mathrm{Glx} / \mathrm{Cr} \\
\mathrm{NAA} / \mathrm{Cr} \\
\mathrm{Lac} / \mathrm{PCr}+\mathrm{Cr} \\
\mathrm{Lip} / \mathrm{PCr}+\mathrm{Cr} \\
\alpha-\mathrm{Glx} / \mathrm{NAA} \\
\mathrm{mI} / \mathrm{NAA} \\
\mathrm{GPC}+\mathrm{PC} / \mathrm{NAA} \\
\beta, \gamma-\mathrm{Glx} / \mathrm{NAA} \\
\mathrm{PCr}+\mathrm{Cr} / \mathrm{NAA}\end{array}$ & $\begin{array}{l}\text { - GAD patients had } \\
\text { significantly lower Cho/Cr } \\
\text { and Cho/NAA ratios in the } \\
\text { DLPFC compared with HC } \\
\text { - Cho ratios were positively } \\
\text { correlated with the brain } \\
\text { activities based on blood } \\
\text { oxygenation } \\
\text { level-dependent signal } \\
\text { change in the DLPFC } \\
\text { - DLPFC volume was } \\
\text { positively correlated with } \\
\text { the ratios of Cho/Cr and } \\
\text { Cho/NAA in GAD patients }\end{array}$ \\
\hline
\end{tabular}




\begin{tabular}{|c|c|c|c|c|c|c|c|c|}
\hline Study & $\begin{array}{c}\text { Sample (age, } \\
\text { mean } \pm \text { S.D.) }\end{array}$ & Gender F/M & Study design & $\begin{array}{l}\text { Psychotropic } \\
\text { medications }\end{array}$ & Method & $\begin{array}{l}\text { Location (voxel } \\
\text { size) }\end{array}$ & $\begin{array}{l}\text { Quantification } \\
\text { and reported }{ }^{1} \mathrm{H} \\
\text { metabolites }\end{array}$ & ${ }^{1} \mathrm{H}$ MRS findings \\
\hline $\begin{array}{l}\text { Moon } \\
\text { et al. } \\
\text { (2015) }\end{array}$ & $\begin{array}{l}\text { GAD patients }= \\
15(35.4 \pm 9.6) \\
\mathrm{HC}=15(38.8 \pm 8.9)\end{array}$ & $\begin{array}{l}\text { GAD patients }=9 / 6 \\
\mathrm{HC}=9 / 6\end{array}$ & Cross-sectional & $\begin{array}{l}\text { Ten patients with } \\
\text { Anxiolytics and/or } \\
\text { antidepressants. } \\
\text { Five patients each were } \\
\text { taking one } \\
\text { psychotropic } \\
\text { medication }\end{array}$ & $\begin{array}{l}\text { Single-voxel } \\
{ }^{1} \mathrm{H}-\mathrm{MRS} \\
\text { with TE }=30 \\
\mathrm{~ms} \text { and MRI } \\
\text { at } 3 \mathrm{~T}\end{array}$ & $\begin{array}{l}\text { DLPFC } \\
\qquad(20 \times 20 \times \\
\left.20 \text { or } 8 \mathrm{~cm}^{3}\right)\end{array}$ & $\begin{array}{l}\text { Lac/NAA } \\
\text { Lip/NAA } \\
\text { Metabolite ratio } \\
\text { NAA } \\
\text { GPC }+ \text { PC } \\
\text { PCr }+ \text { Cr } \\
\text { Ml } \\
\text { Lactate } \\
\text { Lip } \\
\alpha-G l x / P C r+C r \\
\beta, \gamma-G l x / N A A\end{array}$ & $\begin{array}{l}\text { - GAD patients had } \\
\text { significantly lower Cho/Cr } \\
\text { and Cho/NAA ratios in the } \\
\text { DLPFC compared with } \\
\text { HC. } \\
\text { - No significant differences in } \\
\text { other metabolite ratios } \\
\text { between the two groups } \\
\text { - Cho/NAA ratio in GAD } \\
\text { patients was negatively } \\
\text { correlated with the scores of } \\
\text { HAMA and GAD-7 }\end{array}$ \\
\hline $\begin{array}{l}\text { Coplan } \\
\text { et al. } \\
(2014)\end{array}$ & $\begin{array}{l}\text { GAD patients }= \\
29(35.1 \pm 11.9) \\
\mathrm{HC}=22(33.7 \pm 10.4)\end{array}$ & $\begin{array}{l}\text { GAD patients }= \\
18 / 11 \\
\mathrm{HC}=14 / 8\end{array}$ & Cross-sectional & No medication & $\begin{array}{l}\text { Multi-voxel } \\
{ }^{1} \mathrm{H}-\mathrm{MRSI} \\
\text { with TE }= \\
280 \mathrm{~ms} \text { at } 1.5 \\
\mathrm{~T}\end{array}$ & $\begin{array}{l}\text { Left and right } \\
\text { hippocampus } \\
\left(1.13 \mathrm{~cm}^{3}\right)\end{array}$ & $\begin{array}{l}\text { Absolute relative } \\
\text { to water } \\
\text { NAA } \\
\mathrm{PCr}+\mathrm{Cr} \\
\text { GPC }+ \text { PC }\end{array}$ & $\begin{array}{l}\text { - Overweight subjects } \\
\text { exhibited lower NAA levels } \\
\text { in the hippocampus than } \\
\text { normal-weight subjects in } \\
\text { both GAD patients and HC. } \\
\text { - Women overall exhibited } \\
\text { relative elevations of } \\
\text { hippocampal NAA } \\
\text { concentration, compared } \\
\text { with males. } \\
\text { - Lower Cho in the left } \\
\text { hippocampus and higher } \\
\text { Cho in the right } \\
\text { hippocampus in GAD } \\
\text { subjects compared with } \\
\text { HC. } \\
\text { - Reduction in Cr } \\
\text { concentration in GAD }\end{array}$ \\
\hline
\end{tabular}


Table 1. Continued

\begin{tabular}{|c|c|c|c|c|c|c|c|c|}
\hline Study & $\begin{array}{c}\text { Sample (age, } \\
\text { mean } \pm \text { S.D.) }\end{array}$ & Gender F/M & Study design & $\begin{array}{c}\text { Psychotropic } \\
\text { medications }\end{array}$ & Method & $\begin{array}{l}\text { Location (voxel } \\
\text { size) }\end{array}$ & $\begin{array}{l}\text { Quantification } \\
\text { and reported }{ }^{1} \mathrm{H} \\
\text { metabolites }\end{array}$ & ${ }^{1} \mathrm{H}$ MRS findings \\
\hline & & & & & & & & $\begin{array}{l}\text { subjects compared with HC } \\
\text { in the left hippocampus. } \\
\text { - Subjects with BMI } \geq 25 \\
\text { exhibited lower Cr than } \\
\text { subjects with BMI } \leq 25 \text { in } \\
\text { the hippocampus. } \\
\text { - An inverse correlation was } \\
\text { noted in all subjects } \\
\text { between the right } \\
\text { hippocampal NAA and } \\
\text { BMI. } \\
\text { - An inverse linear } \\
\text { correlation was noted in all } \\
\text { subjects between right } \\
\text { hippocampal NAA and } \\
\text { BMI. } \\
\text { - High scores on the PSWQ } \\
\text { predicted low hippocampal } \\
\text { NAA and Cr. } \\
\text { - Both BMI and worry were } \\
\text { independent inverse } \\
\text { predictors of hippocampal } \\
\text { NAA. } \\
\text { - High scores on the PSWQ } \\
\text { predicted low hippocampal } \\
\text { NAA and Cr. Both BMI and } \\
\text { worry were independent } \\
\text { inverse predictors of } \\
\text { hippocampal NAA }\end{array}$ \\
\hline
\end{tabular}

GAD, Generalised anxiety disorder; MRI, Magnetic Resonance Imaging; fMRI, Functional Magnetic Resonance Imaging; MRS, Magnetic Resonance Spectroscopy; MRSI, Magnetic Resonance Spectroscopy Imaging; NAA, N-Acetyl-Aspartate; GPC + PC, Glycerophosphocholine plus Phosphocholine; PCr + Cr, Phosphocreatine plus Creatine; HC, Healthy controls; DLPFC, Dorsolateral prefrontal cortex; ACC, Anterior Cingulate Cortex; SSC, Somatosensory cortex; mPFC, medial prefrontal cortex; BMI, Body mass index; PSWQ, Penn State Worry

Questionnaire. 
GAD. Additionally, from the ${ }^{1} \mathrm{H}$ MRS studies here described emerged that pharmacological treatments positively interact with specific metabolites, especially NAA, within selective brain regions. Therefore, the investigation of brain metabolites could be very effective not only for elucidating the pathophysiology of neuropsychiatric diseases, but also for the identification of more beneficial and targeted pharmacological interventions. Finally, although ${ }^{1} \mathrm{H}$ MRS has been combined with other neuroimaging methods in recent studies (Abdallah et al. 2012a, b, 2013; Moon et al. 2015, 2016b; Moon \& Jeong, 2016a), the evidence are still scarce. However, it is important to point out that the combination of more MRI methods allows the integration of different measures, which might increase the information and consequently the reliability of the findings.

\section{Acknowledgements}

None.

\section{Financial support}

This research received no specific grant from any funding agency, commercial or not-for-profit sectors.

\section{Conflict of interest}

None.

\section{References}

Abdallah CG, Coplan JD, Jackowski A, Sato JR, Mao X, Shungu DC, Mathew SJ (2012a). Riluzole effect on occipital cortex: a structural and spectroscopy pilot study. Neuroscience Letters 530, 103-107.

Abdallah CG, Coplan JD, Jackowski A, Sato JR, Mao X, Shungu DC, Mathew SJ (2012b). A pilot study of hippocampal volume and N-acetylaspartate (NAA) as response biomarkers in riluzole-treated patients with GAD. European Neuropsychopharmacology 23, 276-284.

American Psychiatric Association (2013). Diagnostic and Statistical Manual of Mental Disorders, 5th edn (DSM 5). American Psychiatric Publishing: Arlington, VA.

Brambilla P, Stanley JA, Nicoletti M, Harenski K, Wells KF, Mallinger AG, Keshavan MS, Soares JC (2002). ${ }^{1} \mathrm{H}$ MRS brain measures and acute lorazepam administration in healthy human subjects. Neuropsychopharmacology 26, 546-551.

Brambilla P, Glahn DC, Balestrieri M, Soares JC (2005). Magnetic resonance findings in bipolar disorder. Psychiatric Clinics of North America 28, 443-467.

Brambilla P, Como G, Isola M, Taboga F, Zuliani R, Goljevscek S, Ragogna M, Brondani G, Baiano M, Perini L, Ferro A, Bazzocchi M, Zuiani C, Balestrieri M (2012). White matter abnormalities in the right posterior hemisphere in generalized anxiety disorder: a diffusion imaging study. Psychological Medicine 2, 427-434.

Coplan JD, Fathy HM, Abdallah CG, Ragab SA, Kral JG, Mao X, Shungu DC, Mathew SJ (2014). Reduced hippocampal $\mathrm{N}$-acetyl-aspartate (NAA) as a biomarker for overweight. Neuroimage: Clinical 4, 326-335.

Diwadkar VA, Re M, Cecchetto F, Garzitto M, Piccin S, Bonivento C, Maieron M, D'Agostini S, Balestrieri M, Brambilla P (2017). Attempts at memory control induce dysfunctional brain activation profiles in generalized anxiety disorder: an exploratory fMRI study. Psychiatry Research: Neuroimaging 266, 42-52.

Frizzo MES, Dall'Onder LP, Dalcin KB, Souza DO (2004). Riluzole enhances glutamate uptake in rat astrocyte cultures. Cellular and Molecular Neurobiology 24, 123-128.

Mathew SJ, Mao X, Coplan JD, Smith ELP, Sackeim HA, Gorman JM, Shungu DC (2004). Dorsolateral prefrontal cortical pathology in generalized anxiety disorder: a proton magnetic resonance spectroscopic imaging study. American Journal of Psychiatry 161, 1119-1121.

Mathew SJ, Price RB, Mao X, Smith ELP, Coplan JD, Charney DS, Shungu DC (2008). Hippocampal N-acetylaspartate concentration and response to riluzole in generalized anxiety disorder. Biological Psychiatry 63, 891-898.

Mathew SJ, Price RB, Shungu DC, Mao X, Smith ELP, Amiel JM, Coplan JD (2010). A pilot study of the effects of chronic paroxetine administration on hippocampal $\mathrm{N}$-acetylaspartate in generalized anxiety disorder. Journal of Psychopharmacology 24, 1175-1181.

Moon CM, Jeong GW (2016a). Brain morphological alterations and cellular metabolic changes in patients with generalized anxiety disorder: a combined DARTEL-based VBM and (1)H-MRS study.

Moon CM, Kang HK, Jeong GW (2015). Metabolic change in the right dorsolateral prefrontal cortex and its correlation with symptom severity in patients with generalized anxiety disorder: proton magnetic resonance spectroscopy at 3 Tesla. Psychiatry and Clinical Neuroscience 69, 422-430.

Moon CM, Sundaram T, Choi NG, Jeong GW (2016b). Working memory dysfunction associated with brain functional deficits and cellular metabolic changes in patients with generalized anxiety disorder. Psychiatry Research 254, 134-144.

Raparia E, Coplan JD, Abdallah CG, Hof PR, Mao X, Mathew SJ, Shungu DC (2016). Impact of childhood emotional abuse on neocortical neurometabolites and complex emotional processing in patients with generalized anxiety disorder. Journal of Affective Disorder 190, 414-423.

Stanley JA (2002). In vivo magnetic resonance spectroscopy and its application to neuropsychiatric disorders. Canadian Journal of Psychiatry 47, 315-326.

Stanley JA, Vemulapalli M, Nutche J, Montrose DM, Sweeney JA, Pettegrew JW, MacMaster FP, Keshavan MS (2007). Reduced N-acetyl-aspartate levels in schizophrenia patients with a younger onset age: a single-voxel $1 \mathrm{H}$ spectroscopy study. Schizophrenia Research 93, 23-32.

Strawn JR, Chu WJ, Whitsel RM, Weber WA, Norris MM, Adler CM, Eliassen JC, Phan KL, Strakowski SM, DelBello MP (2013). A pilot study of anterior cingulate 
cortex neurochemistry in adolescents with generalized anxiety disorder. Neuropsychobiology 67, 224-229.

Terlevic R, Isola M, Ragogna M, Meduri M, Canalaz F, Perini L, Rambaldelli G, Travan L, Crivellato E, Tognin S, Como G, Zuiani C, Bazzocchi M, Balestrieri M, Brambilla P (2012). Decreased hypothalamus volumes in generalized anxiety disorder but not in panic disorder. Journal of Affective Disorder 146, 390-394.

Wang JW, David DJ, Monckton JE, Battaglia F, Hen R (2008). Chronic fluoxetine stimulates maturation and synaptic plasticity of adult-born hippocampal granule cells. Journal of Neuroscience 28, 1374-1384. 